DE92 0.16281

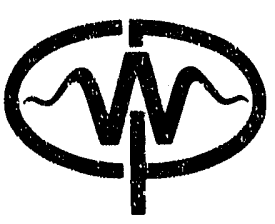

\title{
Finite-Difference Migration to Zero Offset
}

\author{
Jianchao Li
}

Center for Wave Phenomena

Colorado School of Mines

Golden, Colorado 80401

(303) 273-355t 


\title{
Finite-Difference Migration to Zero Offset
}

\author{
Jianchao Li
}

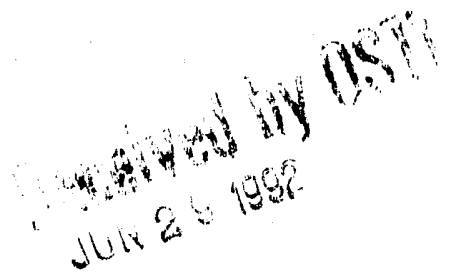

\begin{abstract}
Migration to zero offset (MZO), also called dip moveout (DMO) or prestack partial migration, transiurms prestack offset seismic data into approximate zerooffset data so as to remove reffection point smear and obtain quality stacked results over a range of reflector dips. MZO has become an important step in standard seismic data processing, and a variety of frequency-wavenumber $(f-k)$ and integral MZO algorithms have been used in practice to date.

Here, I present a finite-difference MZO algorithm applied to normal-moveout (NMO)-corrected, common-offset sections. This algorithm employs a traditional poststack 15-degree finite-difference migration algorithm and a special velocity function rather than the true migration volocity. This paper shows results of implementation of this $\mathrm{MZO}$ algorithm when velocity varies with depth, and discusses the possibility of applying this algorithm to cases where velocity varies with both depth and horizontal distance.
\end{abstract}

\section{INTRODUCTION}

When reflectors are dipping, common-midpoint (CMP) gathers recorded at the Earth's surface are no longer common-reflection-point (CRP) records. The reflections in a CMP gather, in fact, come from different reflection points, a phenomenon called reflection point smear or dispersal. Although NMO correction can align the reflections that come from horizontal reffectors, it fails to correct those reflections that come from reflectors with a range of dips. As a result, CMP stack after NMO correction will attenuate non-aligned reflections. The reason for this failure, roughly speaking, is that NMO correction is a $1-D$ process, while reflection-point smear is a $2-D$ problem; and $1-\mathrm{D}$ processes cannot solve $2-\mathrm{D}$ problems. 
One way to ensure CRP stacking of seismic data is the application of full prestack migration (preferably full prestacli depth migration) to offset clata. In principle, this is an ideal way because it can directly position reflections in offset data to their original reflection points. Generally speaking, however, full prestack migration is computationally expensive.

A second approach which acemplishes CIRP stacking in an indirect and approximate manner, consists of four steps: NMO correction, migration to zero offset (MZO), stacking, and poststack migration. The result after the application of these four steps is approximately equivalent to that obtained by using full prestark migration. This approach, in gencral, is much more practical than full prestack migration.

$\mathrm{MZO}$, also (alled dip moveout. (DMO) or prestack partial migration, is a key step) in the second approach mentioned above. As its name implies, this 2-D prestack process transforms prestack offsct data so that they appear like true zero-offset data.

Many papers have been published on the two most commonly used MZO approaches: frequency-wavenumber $(f-h)$ MZO) and integral MZO (Hale, 1988). In contrast, only a few papers on finite-diffenence MZO have been published so far. Finte-difference algorithms play an inportant role in conventional migration methorls because they ean readily handle velocity variation. Since MZO is a form of migration, finite-difference algorithms shonld have application there, as well.

Yilmaz and Clachlout (1980) proposed two finite-rlifference MZO algorithms from analysis of the domble square-root equation. Bolondi, ot al. (1982) and Salvador and Savelli (1982) presented another finite-differenee MZO algorithm, based on the concept of offset continuation. These algorithms have generally low efficieney and low aceuracy for landling large-offset seismice sections atud reflections from steep reffectors.

Here, I present a finite-rliferene $\mathrm{MZO}$ algorithm applied to NMO-corrected commonoffiset sections. Tests show the offoretiveness of this algorithm for handling velocity variations, large offsets, and reflections from steep inderfaces.

\section{DERIVATION OF MZO VELOCITY}

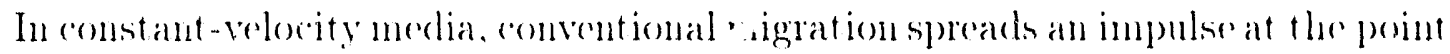
$\left(. r=0, t_{0}\right)$ in the stacked section to the impulse-response trajertory defined by

$$
x^{2}+2^{2}=\left(1 t_{0} / 2\right)^{2}
$$

where $x$ is the CXP coordinate $:$ is depth, $t_{0}$ is zero-offset reftertion time, and $"$ is the medimu velocity. Equation (1) deseribes a circle with radius it $/ 2$ on the $x$ - : plane, the migrated-position domain. If we let $\tau \equiv 2: / 1$ denote vertical time. on time-cequivalent depth, equation (1) beromes

$$
\frac{x^{2}}{\left(1 t_{0} / 2\right)^{2}}+\frac{\tau^{2}}{t_{0}^{2}}=1
$$


This is an elliptic equation on the $x-\tau$ plane, whose two axes are $v t_{0}$ and $2 t_{0}$, respectively. That is, the impulse response of conventional migration is a circle on the $x-z$ plane, while it is an ellipse on the $x-\tau$ plane.

Now, consider the behavior of MZO. The MZO ellipse, after NMO correction, is defined by

$$
\frac{x^{2}}{h^{2}}+\frac{t_{0}^{2}}{t_{n}^{2}}=1,
$$

where $t_{n}$ is the time after NMO correction, and $h$ is half the source-receiver offset (Hale, 1988). If we define a velocity $v$ such that

$$
h \equiv i t_{n} / 2
$$

OH

$$
i \equiv 2 h / t_{n},
$$

and define a depth cuantity

$$
\vdots \equiv i t_{0} / 2
$$

then equation $(3)$ becomes

$$
r^{2}+z^{2}=\left(i t_{n} / 2\right)^{2} .
$$

defining a circle with radius it $/ 2$. That is, the impulse response of $M Z()$ after $N M O$ correction is an cllipse whose two axes are $2 h$ and $2 t_{n}$, resperetively, on the $x$ - $t_{0}$ plane, while it is a circle on the ri-z plane.

Comparing equations (3) and (5) whth equations (1) and (2), we find that if the velocity $i$ is used instead of $v$, then any conventional migration algorithm can be used to perform MZO processing; with this choice, the impulse response of conventional migration is just the MZO) cllipse defined by equation (3). I call $i$, defined by equation (4), the MZO) velocity. Aside from use of a different velocity, the other difference between conventional migration and $\mathrm{MZO}$ is that the input to conventional unigration is $P\left(x, t_{0}\right)$ and the ontput is $P(x, \tau)$, while the input to $\mathrm{MZO}$ is $P\left(x, t_{n}\right)$ and the output is $P\left(x, t_{0}\right)$. Here, $P$ denotes seismic data.

\section{FINITE-DIFFERENCE MZO}

\section{Finite-difference MZO for constant velocity}

In the previous section, we defined MZO) velocity in constant-velocity media and showed that conventional migration algorithms can be used to perform MZO. Let 
us now consider how MZO can be implemented with a traditional poststack, 15degrec finite-difference migration algorithm (Claerbout, 1985). Two reasons for this choice are: (1) among all finite-difference migration schemes, this one has the highest. computational efficiency, and (2) examples and analysis in later sections show that, for MZO, this algorithm has sufficiont accuracy and ability to handle large-offset data where subsurface reflectors are steep. However, when using a finite-difference algorithm to perform MZO, we must address a practical problem. According to the derivation of $\mathrm{MZO}$ velocity, when we compute the response of an impulse at the point $\left(x, t_{n}\right), i$ should be a constant from the time 0 to $t_{n}$. This problem, which exists with finite-difference MZO apperoacless, can be casily and exactly solved in non-recursive integral algorithms, but camnot be exactly solved in finite-difference algorithms, which are recursive processics.

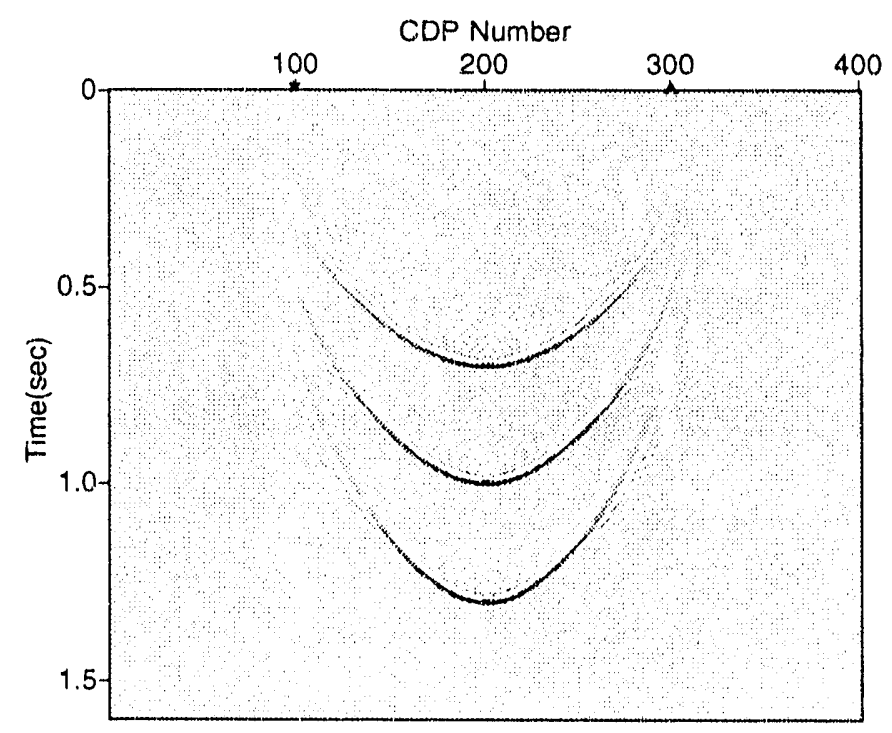

FIG. 1. Inpulse responses of finitr-differenee MZO with velocity in. In this test the CMP interval is $10 \mathrm{~m}$, and offiset $=2 \mathrm{~km}$.

The impulse responses of finite-differener MZO in Figure 1 show the result of using the velocity defined by efuation (4). The distance between the intressections of an impulse response with the surface is greater than $2 \mathrm{~h}$, indicating that the velocity us a to obtain this figme is too large. The tests I have douse suggest that we cain approximately solve this problem by replacing i", as given above, hy

$$
=2 h /\left[\gamma_{n} s\left(t_{0}\right)\right] .
$$

$\ddot{z}$ is the MZO velocity we nse in finite-difference MZO muler the constant-merliumvelocity assumption. I find that when I choose s(to) that decreases lincarly from about 1.1 at the minimm processing time to 1.0 at the maximum processing time, the impulse responses are sefuered to approximately the correct shapes. With this 
slight adjustment of velocity, the finite-difference $M Z O$ algorithm can bo readily implemented by modifying existing 15 -degree finite-difference migration code.

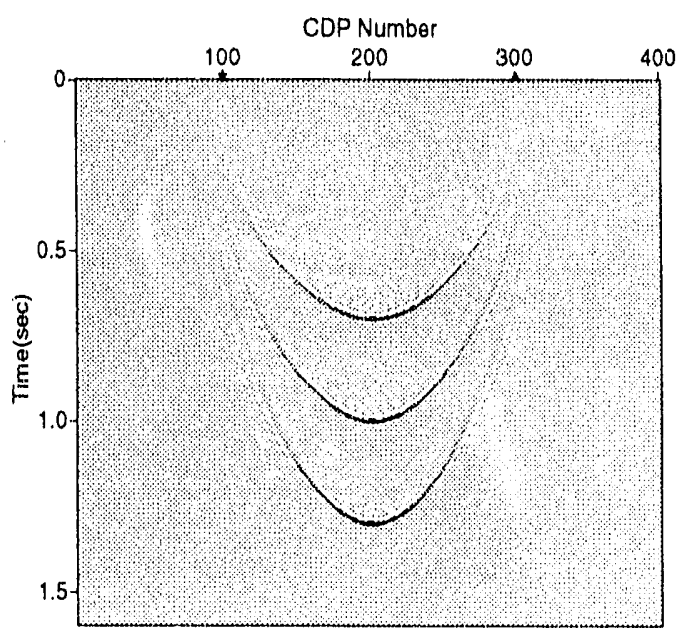

(a)

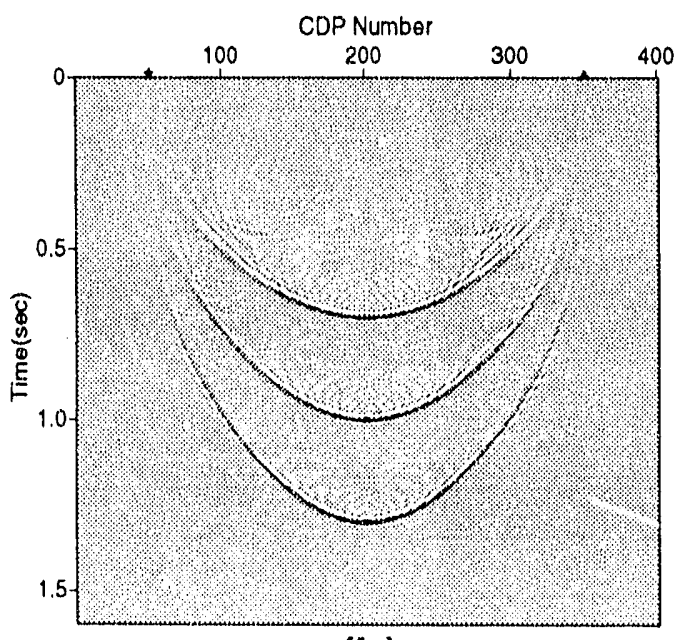

(b)

FIG. 2. Impulse responses of finite-difference MZO for constant velocity. In this test, the CMP interval is $10 \mathrm{~m}$. (a) Offset $=2 \mathrm{~km}$. (b) Offset $=3 \mathrm{~km}$.

Figure 2 shows impulse responses of finite-difference MZO obtained by using the velocity defined by equation (6) with offset of $2 \mathrm{~km}$, in Figure $2 \mathrm{a}$, and $3 \mathrm{~km}$, in Figure 2h. In both the cases, impulse responses are lialf ellipses and have a tendency to intersect the surface at the shot and receiver points. We sense from this figure that although a so-called 15-degree approximate algorithm is used, we have achieved enongh acruracy for both the large offsets and the large dips. These results suggest that when finite-difference algorithms are used to implement conventional migration and $\mathrm{MZO}$, the concept of dip-linited accuracy differs for conventional migration and $\mathrm{MZO}$.

\section{Finite-difference $\mathrm{MZO}$ for depth-variable veiocity}

MZO for depth-variable velocity is often called $V(z)$ MZO. Although velocity variation is often ignored in $\mathrm{MZO}$ processing, and constant-velocity $\mathrm{MZO}$ processing is often used in areas where velocity is known to vary, many authors (e.g., Hale. and Artley, 1991; Artley, 1991: Witte, 1991) have recognized the need to include velocity variation in $M Z O$ processing and have attempted to improved the accuracy of $\mathrm{MZO}$ approaches when velocity varies with depth. However, generally speaking, it is relatively costly for MZO) to landle velocity variations exactly. By introducing Hale's $\gamma\left(t_{n}\right)$ factor (Hale, 1988) in the definition of $\mathrm{MZO}$ velocity, we can extend the above finite-difference $\mathrm{MZO}$ algerithm of that it can approximately handle velocity variations with depth. Hale (1988) has shown that, for depth-variable velocity, the $\mathrm{MZO}$ ellipse defined by equation (3) should be modified as 


$$
\frac{x^{2}}{\gamma\left(t_{n}\right) h^{2}}+\frac{t_{0}^{2}}{t_{n}^{2}}=1
$$

where

$$
\begin{gathered}
\gamma\left(t_{n}\right)=\frac{3 v_{4}^{4}\left(t_{n}\right)}{2 v_{2}^{4}\left(t_{n}\right)}-\frac{t_{n}}{v_{2}\left(t_{n}\right)} \frac{d v_{2}}{d t_{n}}-\frac{1}{2}, \\
v_{2}\left(t_{n}\right)=\left[\frac{1}{t_{n}} \int_{0}^{t_{n}} v^{2}(s) d s\right]^{1 / 2},
\end{gathered}
$$

and

$$
v_{4}\left(t_{n}\right)=\left[\frac{1}{t_{n}} \int_{0}^{l_{n}} v^{4}(s) d s\right]^{1 / 4}
$$

If we define

$$
\bar{v} \equiv 2 \sqrt{\gamma} h / t_{n}
$$

and

$$
\bar{s} \equiv \bar{v} t_{0} / 2
$$

then we obtain

$$
x^{2}+\bar{z}^{2}=\left(\bar{v} t_{n} / 2\right)^{2}
$$

from equation (7). This is also the equation of a circle. Now, we see that the difference between the cases of constant and depth-variable velocity is only in the different definitions of MZO velocity. Moreover, since $\gamma$ is dependent on $t_{n}$ only, MZO velocity $\bar{v}$, defined by equation (9), is still a function of $t_{n}$, just as is $\tilde{v}$ in the constant-velocity case. So, finite-difference MZO for depth-variable velocity can also be implemented in the same way as in the constant-velocity case. As in equation (6), we also incorporate the $s\left(t_{0}\right)$ factor into the definition of $V(z) \mathrm{MZO}$ velocity, equation $(9)$,

$$
\overline{\bar{v}}=2 \sqrt{\gamma} h /\left[t_{n} s\left(t_{0}\right)\right]
$$

\section{Finite-difference $M Z O$ for $(x, z)$-variable velocity}

$V(x, z) \mathrm{MZO}$, migration to zero offset for $(x, z)$-variable-velocity media, is a new topic. Van der Schoot, et al. (1989), Popovici (1990), and Hsu (1991) have used modeled traveltime calculation to address this topic. Although the MZO algorithms 
discussed above are not theoretically suitable to the laterally variable velocity case, the finite-difference algorithm we adopt provides a possible mumerical way for MZO to approximately handle velocity variations in the lateral direction. As is known, neither NMO correction before MZO nor time migration after MZO can exactly deal with laterally variable velocity in theory. In practice, however, these processes handle this issue through lateral interpolation of velocities defined at a number of CMPs in a seismic section and usually can produce acceptable results. For MZO in $v(x, z)$ media with moderate lateral velocity variation, we simply use the $v(z)$ approach developed above with slowly changing velocity along a line, much as is done in conventional NMO and time-migration processing. Since MZO is relatively insensitive to velocity variation, errors in this kind of $\mathrm{MZO}$ processing are expected to be smaller than the errors that arise when such a simple approach to lateral variation is taken in NMO correction and time migration.
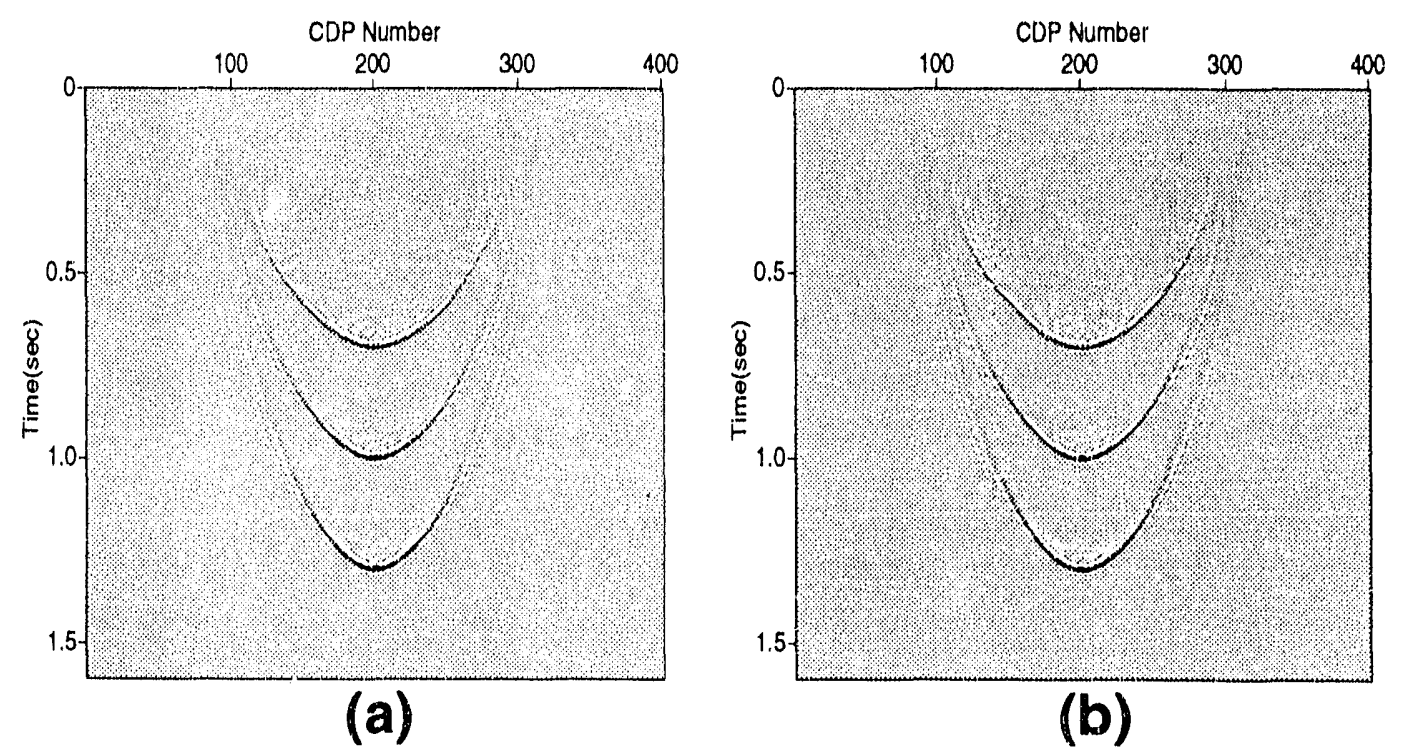

FIG. 3. Impulse responses of finite-difference MZO for (a) the $V(z)$ case and $(b)$ the $V(x, z)$ case. The CMP interval is $10 \mathrm{~m}$.

Figure 3a shows the impulse responses of $V(z) \mathrm{MZO}$ for offset $=2 \mathrm{~km}$. In this test, velocity linearly increases from $3 \mathrm{~km} / \mathrm{s}$ at $0.1 \mathrm{~s}$ to $4 \mathrm{~km} / \mathrm{s}$ at $1.4 \mathrm{~s}$. Comparing Figure 3a with Figure 2a, we sec that volocity variation with depth causes the impulse response to narrow slightly. This is consistent with what Hale and Artley (1991) have described. Figure $3 b$ shows the impulse responses of approximate $V(x, z)$ MZO. In this test, I use the same parameters as in Figure $3 \mathrm{a}$, as well as a 0.5 per kilometer linear increase of velocity in the $x$-direction. We see only minor asymmetry between the left and right branches of the impulse responses in this figure. This suggests that the influence of velocity variation in the lateral direction may not be as significant in $\mathrm{MZO}$ as in conventional migration. 


\section{SYNTHETIC DATA EXAMPLES}

Figure 4 shows a geological model containing nine horizontal reflector segments and five dipping reflector segments, with dips range from 30 to 90 degrees in 15-degree increments. For the synthetic seismic datia, the velocity used increases linearly with depth $z$, according to $v(z)=1.5+0.8 z \mathrm{~km} / \mathrm{s}$, and the CMP interval is $5 \mathrm{~m}$.

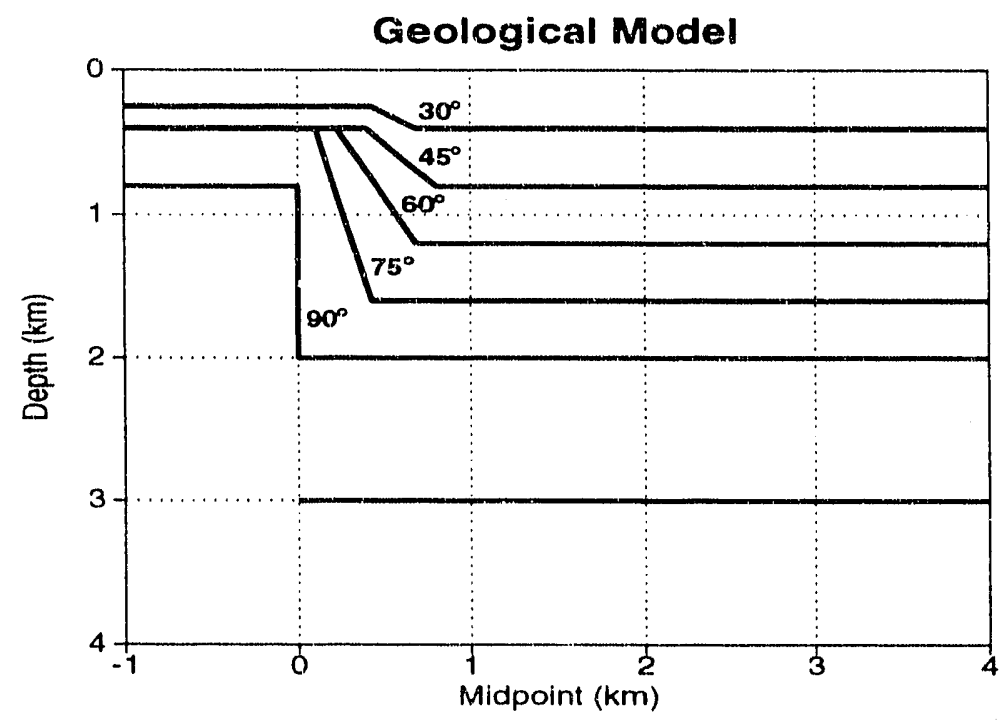

Fig. 4. Geological model used to generate synthetic seismic data.

Four sections obtained after different processings are shown in Figure 5. Figure 5a is a zero-offset synthetic section. The goal of applying $\mathrm{MZO}$ is to transform offset data into this form of data. The dashed line in this figure marks the location of the CMP gather shown in Figure 6, and the box shows the region detailed in Figures 5b, $5 \mathrm{c}$ and $5 \mathrm{~d}$. Figure $5 \mathrm{~b}$ is a detail of the stacked section obtained by simply stacking ten NMO-corrected common-offset sections, whose offsets range from 0.1 to $1.9 \mathrm{~km}$. We see that the dipping reflections, especially the reflection from the 90-degree reflector, have been attenuated, and some noise, due to out-of-phase stacking, appears among the dipping reflections.

A detail of the stacked result after NMO and constant-velocity MZO corrections is plotted in Figure 5c. Compared with Figure 5b, the stacking quality for dipping reflections in this figure has been significantly improved, but the energy of the dipping reflections is still weaker. Figure 5d shows a detail of the stacked result after NMO and $V(z) \mathrm{MZO}$ corrections. In $V(z) \mathrm{MZO}$ processing, we used the known rms velocity $v_{2}\left(t_{n}\right)$ for the model. We see from this figure that the stacking quality for dipping reflections, especially the reflection from the 90-degree reflector, has been further significantly improved, and all reflections have amplitudes close to those in the zerooffset section shown in Figure 5a. For comparison, we have used the identical stacking and plotting parameters to get four figures. 


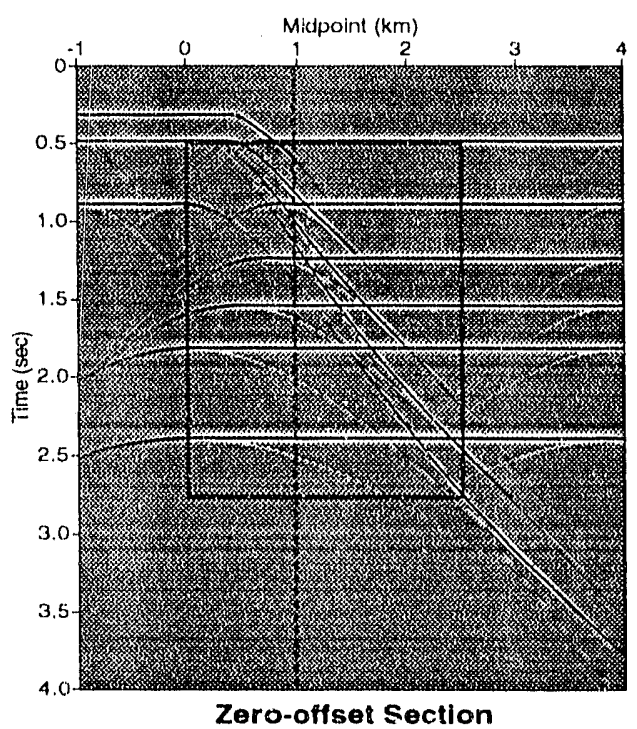

(a)

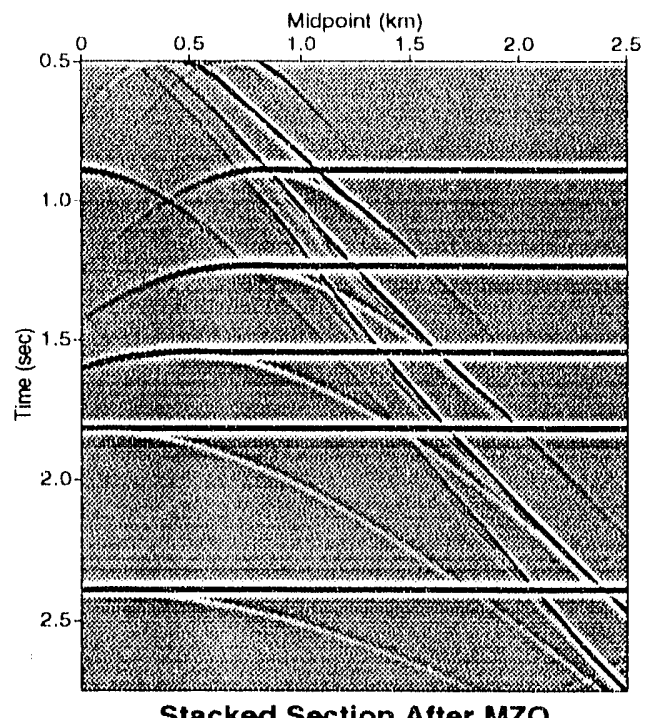

(c)

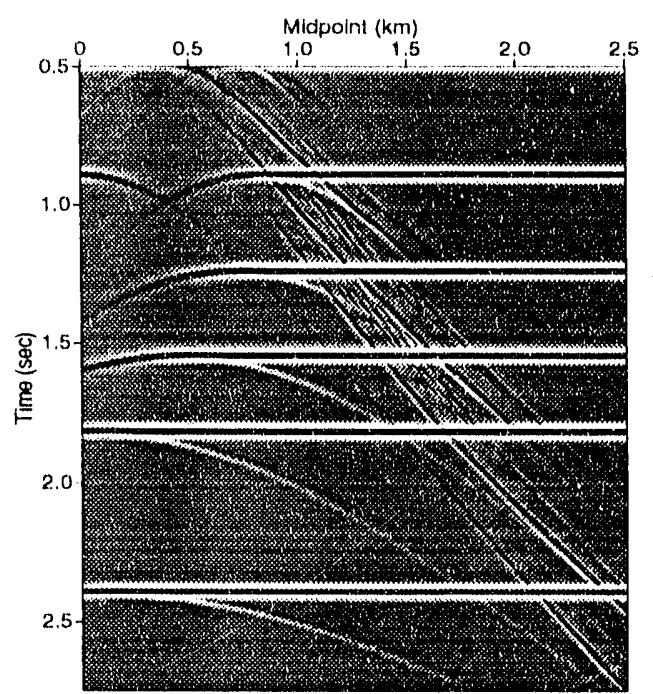

Stacked Section After NMO

(b)

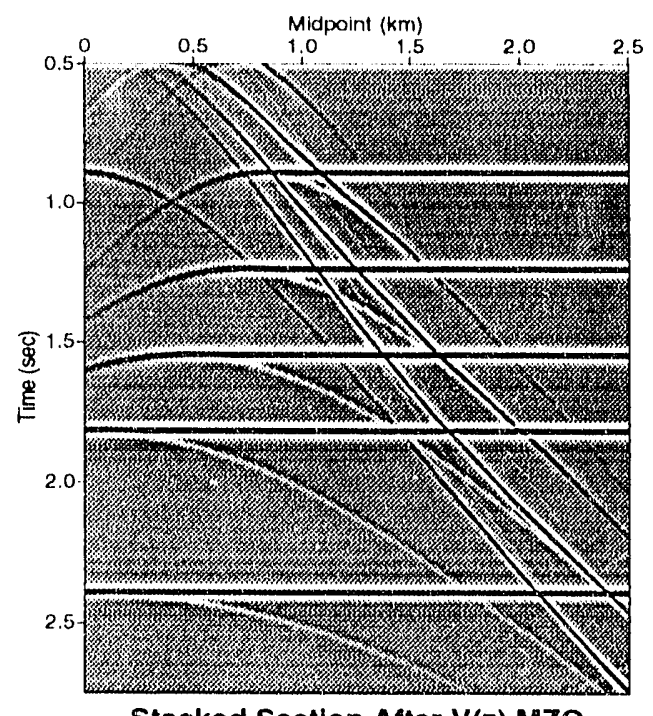

Stacked Section After V(z) MZO

(d)

FIG. 5. (a) Zero-offset synthetic section for the geological model shown in Figure 4. The rlashed line marks the location of the CMP gather shown in Figure 6. The box shows the location of (b), (c) and (d). (b) Detail of stacked section after NMO correction. (c) Detail of stacked section after NMO and constant-velocity MZO corrections. (d) Detail of stacked section after NMO and $V(z)$ MZO corrections. 


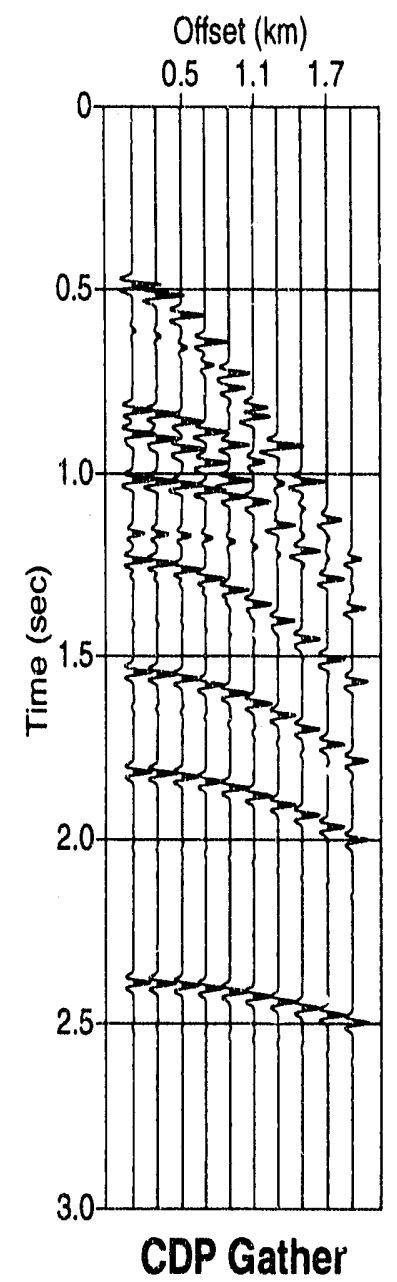

(a)

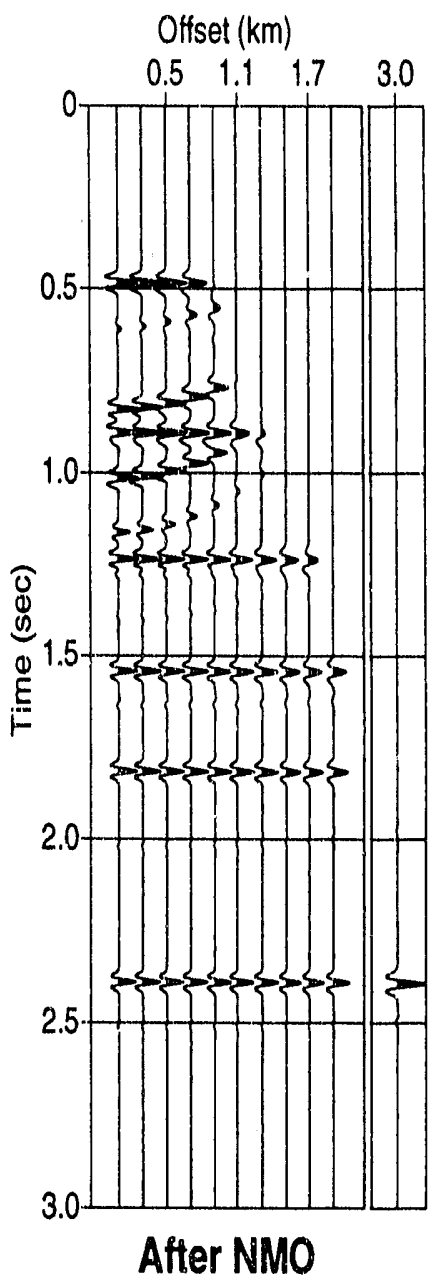

(b)

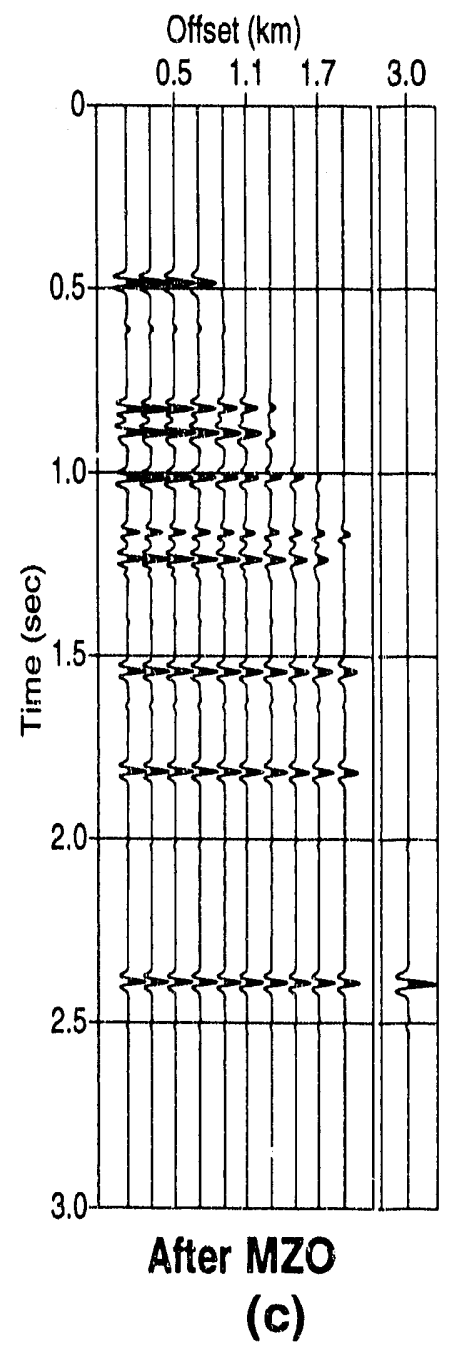

(c)

FIG. 6. (a) CMP gather for the model showed in Figure 4. (b) After NMO correction. (c) After $V(z)$ MZO. 


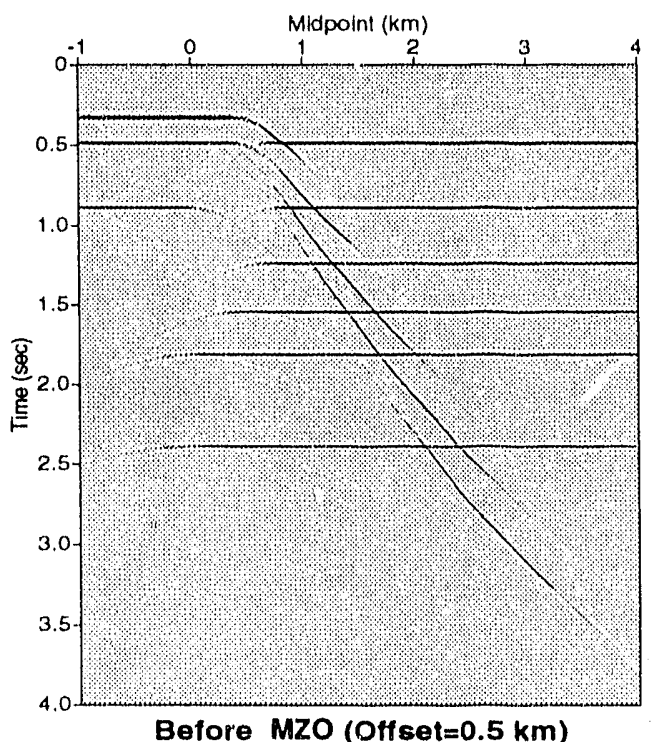

(a)

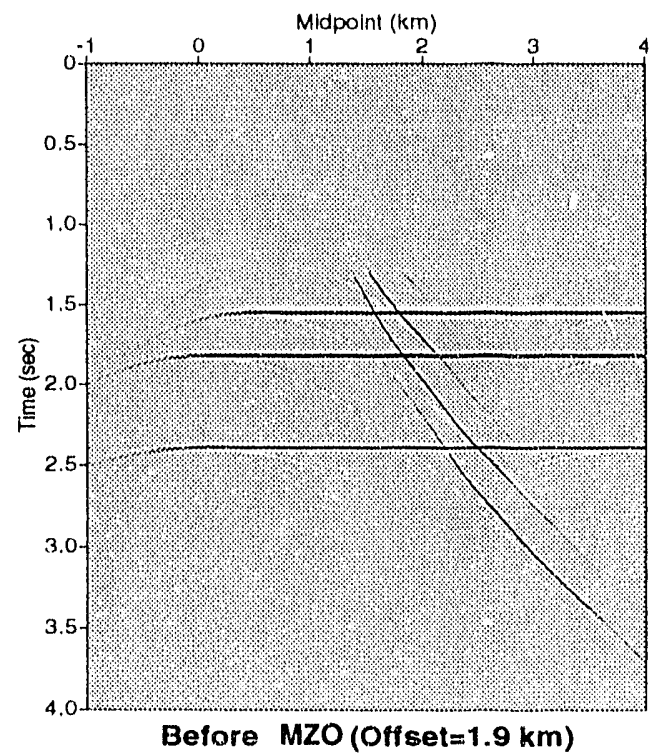

(c)

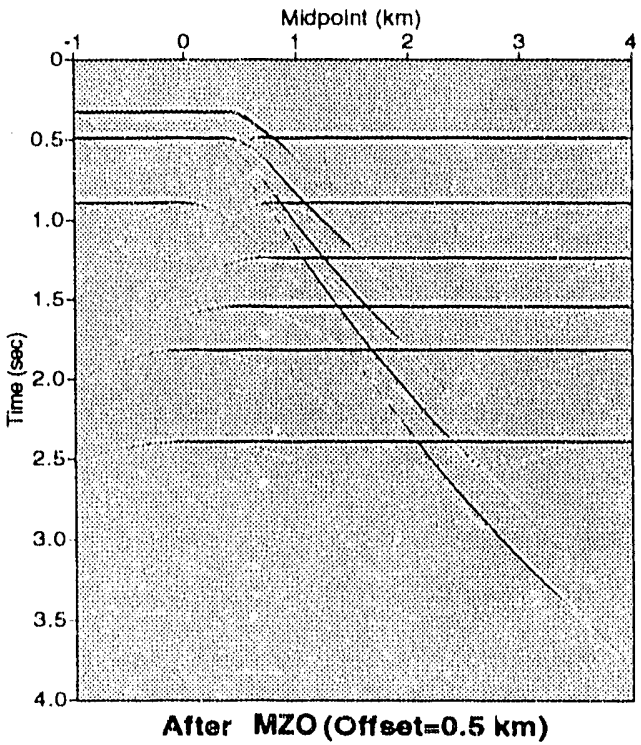

(b)

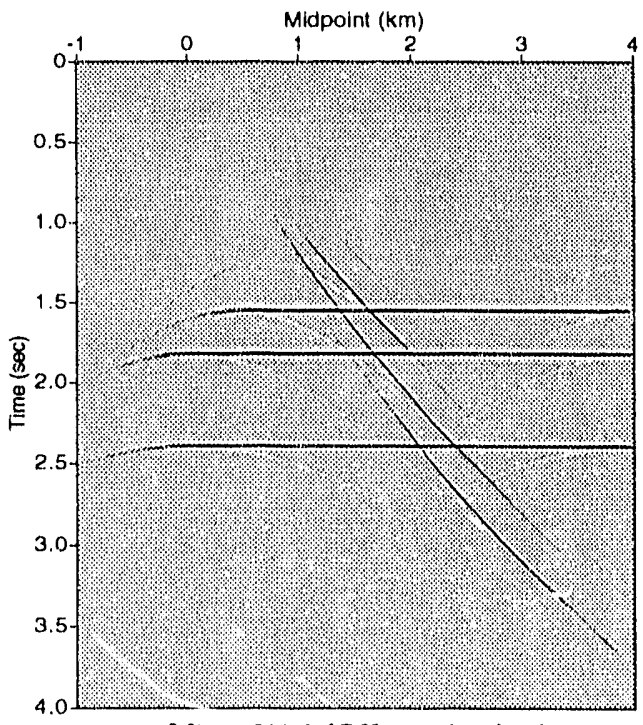

After MZO (Offset $=1.9 \mathrm{~km}$ )

(d)

FIG. 7. Comparison among the common-offset sections before and after MZO. 
Figure 6 shows the reason why the stacked section after $V(z) \mathrm{MZO}$ correction is better than the stacked sections after NMO and constant-velocity MZO corrections. One of the CMP gathers from the model shown in Figure 4 is plotted in Figure 6a. This gather contains six events from horizontal reflectors, five events from dipping reflection segments, and several weak diffraction events from the structural corners. The result of NMO correction applied to the CMP gather of Figure 6a is plotted in Figure 6b. Although the events corresponding to the horizontal reflectors in this gather have been well aligned, some problems appear: (1) the reflections from the dipping reflectors have been over-corrected; (2) the diffractions have also been overcorrected; and (3) anomalously, the event at about 0.6 s corresponding to the 30 degree dipping reflector has amplitude increasing with offset. Obviously, if this NMOcorrected CMP gather is stacked, the poorly aligned events from the dipping reflectors and the diffraction events will be at fenuated.

MZO attempts to solve all these problems. Figure 6e shows the result of the finite-difference $V(z) \mathrm{MZO}$ correction of Figure $6 \mathrm{~b}$. All events that had been overcorrected by NMO in Figure 6b have been aligned back to their "correct" positions with only slightly visible errors, even for the trace with offset of $3 \mathrm{~km}$. As a result, stacking this CMP gather must enhance all events. Furthermore, amplitude values of the event from the 30-degree dipping reflector now decrease with offset, as do those from the other reffectors. In this test, I took the function $s\left(t_{0}\right)=1.11$ at the minimum processing time. Here. I have not shown the constant-velocity MZO-corrected CMP gather. The constant-velocity MZO did not align all the over-corrected events as well as did the $V^{\prime}(z) \mathrm{MZO}$ (Hale and Artley 1991). So, the stacking quality after the constant-velocity MZO correction is not as good as that after $V(z)$ MZO correction.

Figure $z$ offers a comparison among common-offset sections before and after MZO. This figure demonst rates two points: (1) .MZO moves dipping reflections only a small amount in both the horizont al and the vertical directions: and (2) alt hough a 15 -degree approximate algorithm is used. unlike conventional migration, finite-difference MZO does not generate dispersion or other artifacts even for the reflection corresponding to the 90-degree reflector. These facts again illustrate that the concept of limitation of dips differs for conventional migration and $\mathrm{MZO}$.

\section{CONCLUSION}

From the similar kincmaties of conventional migration and . MZO, I have shown that conventional time migration algorithms, with an approximate choice of $M Z O \mathrm{~V}$ locity, can be used to implenent $\mathrm{MZO}$ processing. Finite-difference $\mathrm{NZO}$ algorithms for constant-velocity and depth-variable velority have been proposed in this paper, with a suggestion for extending their application to $(x, r, y)$ media.

The inpulse response tests show the correctuess of the finite-difference MZO algorithms. Successful application of finite-difference MZO to synthetic seisnic data for a geological model containing steep reffectors, up to 90-degree dip, demonstrates 
the effectiveness of finite-difference MZO in handling steep events, large offsets and depth-variable velocity.

Obviously, the finite-difference MZO discussed in this paper is only an approximate method. There are still unsolved problems. For example, finite-difference MZO cannot take into account the two distinct branches of $V(z)$ MZO impulse responses (Artley, 1991). The computational cost of finite-difference MZO is comparable to that of poststack finite-difference migration, but its efficiency is lower than that of $f-k \mathrm{MZO}$.

\section{ACKNOWLEDGEMENTS}

Thanks to Dr. Ken Larner for his critical reading and editing of my writing. This work was partially supported by the Consortium at the Center for Wave Phenomena, Colorado School of Mines and by the United States Department of Energy, Graint Number DE-FG02-89ER14079. (This support does not constitute an endorsement by $\mathrm{DOE}$ of the views expressed in this paper.)

\section{REFERENCES}

Artley, C., 1991, Dip-moveout processing for cepth-variable velocity : presented at the 61th Annual International SEG Meeting, Houston, Texas.

Bolondi, G., Loinger, E , and Rocca, F., 1982, Offset continuation of seismic sections: Geophysical Prospecting, 30, 813-828.

Claerbout, J. F., 1985, Imaging the carth's interior: Blackwell Scientific Publications. Hale, D., 1988, Dip moveout processing - course notes: Soc. Expl. Geophys.

Hale, D. and Artley, C., 1991, Squeezing dip movcout for depth-variable velocity: CW-112, Colorado School of Mines.

Hsu, Ching-Hsiang, :991, Kinematics of dip-moveout for variable media: in CWP. 107, Colorado School of Mines.

Popovici, A. M., 1990, Kinematics of prestack partial migration in variable velocity media: presented at the 60th Annual International SEG Meeting, San Fransico, California.

Salvador, L. and Savelii, S., 1982, Offset continuation for seismic stacking: Geophysical Prospecting, 30, 829-849.

Van der Schoot, A., Duyndam, A. J., Wapenarr, C. P. A., and Berkhout, A. J., 1989, Common-point-stacking: A macro-model driven approach to DMO: presented at the 59th Annual International SEG Meeting, Dalas, Texas.

Witte, D., 1991, Dip moveout in vertically varying media: presented at the 61th Annual International SEG Meeting, Houston, Texas.

Yilmaz, O. and Clacrbout, J. F., 1980, Prestack partial migration: Geophysics, 45, 1753-1779. 
DATE

FILMED 8127192 


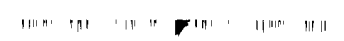

